\title{
A meta-analysis of genome-wide association studies using Japanese and Taiwanese has revealed novel loci associated with gout susceptibility
}

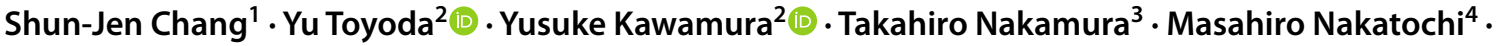

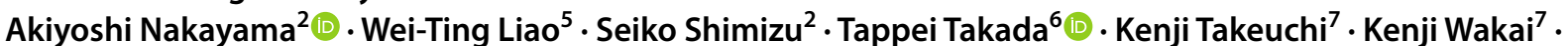 \\ Yongyong Shi ${ }^{8,9} \cdot$ Nariyoshi Shinomiya ${ }^{2} \mathbb{1 0}$. Chung-Jen Chen $^{10}$. Changgui $\mathrm{Li}^{11} \cdot$ Yukinori Okada $^{12,13,14,15}$. \\ Kimiyoshi Ichida ${ }^{16} \cdot$ Hirotaka Matsuo ${ }^{2}$ (]) for Japan Gout Genomics Consortium (Japan Gout)
}

Received: 29 November 2021 / Accepted: 21 December 2021 / Published online: 15 January 2022

(c) The Author(s) 2022

To the Editor,

We have recently identified four novel genomic loci influencing gout susceptibility at the genome-wide significance level $\left(P<5.0 \times 10^{-8}\right)$ via genome-wide association study (GWAS) meta-analyses of clinically defined gout with more finely differentiated subtypes in Japanese cohorts [1]. However, there are many loci that are inconclusive but suggestive of an association with the risk of gout. This prompted us to carry out a meta-analysis using previous gout GWASs of the

Shun-Jen Chang, Yu Toyoda and Yusuke Kawamura contributed equally to this study.

The members of Japan Gout Genomics Consortium (Japan Gout) are listed in acknowledgements.

Hirotaka Matsuo

hmatsuo.ndmc@gmail.com

1 Department of Kinesiology, Health and Leisure Studies, National University of Kaohsiung, Kaohsiung, Taiwan

2 Department of Integrative Physiology and Bio-Nano Medicine, National Defense Medical College, 3-2 Namiki, Tokorozawa, Saitama 359-8513, Japan

3 Laboratory for Mathematics, National Defense Medical College, Saitama, Japan

4 Public Health Informatics Unit, Department of Integrated Health Sciences, Nagoya University Graduate School of Medicine, Aichi, Japan

5 Department of Biotechnology, College of Life Science, Kaohsiung Medical University, Kaohsiung, Taiwan

6 Department of Pharmacy, The University of Tokyo Hospital, Tokyo, Japan

7 Department of Preventive Medicine, Nagoya University Graduate School of Medicine, Aichi, Japan
Japanese [1] and the Taiwanese [2] populations. Integration of the results allowed us to focus on 11 SNPs $\left(P<1.0 \times 10^{-5}\right.$ in the Japanese populations in our previous study [1]), for which information for conducting a meta-analysis was available (Supplementary Table S1). As described below, we successfully identified, for we believe the first time, two loci associated with the risk of gout at a genome-wide level of significance.

Details of the study participants, including a total of 3800 gout cases and 6625 controls (Japanese: 3053 cases

8 Bio-X Institutes, Key Laboratory for the Genetics of Developmental and Neuropsychiatric Disorders (Ministry of Education), Shanghai Jiao Tong University, Shanghai, China

9 Affiliated Hospital of Qingdao University and Biomedical Sciences Institute of Qingdao University (Qingdao Branch of SJTU Bio-X Institutes), Qingdao University, Qingdao, China

10 Department of Internal Medicine, Kaohsiung Medical University Hospital, Kaohsiung, Taiwan

11 Department of Endocrinology and Metabolism, The Affiliated Hospital of Qingdao University, Qingdao, China

12 Department of Statistical Genetics, Osaka University Graduate School of Medicine, Osaka, Japan

13 Laboratory of Statistical Immunology, Immunology Frontier Research Center (WPI-IFReC), Osaka University, Osaka, Japan

14 Integrated Frontier Research for Medical Science Division, Institute for Open and Transdisciplinary Research Initiatives, Osaka University, Osaka, Japan

15 Laboratory for Systems Genetics, RIKEN Center for Integrative Medical Sciences, Yokohama, Japan

16 Department of Pathophysiology, Tokyo University of Pharmacy and Life Sciences, Tokyo, Japan 
and 4554 controls; Taiwanese: 747 cases and 2071 controls), were described previously [1,2]. Regarding the 11 SNPs potentially associated with gout, we obtained association summary statistics data of the SNPs from the published two GWASs and combined them. Our meta-analysis of gout revealed genome-wide significant associations of rs 16998073-T (T/A: major allele is A) [intergenic between PR/SET Domain 8 (PRDM8) and fibroblast growth factor 5 (FGF5)] and rs10847689-C (C/T: major allele is T) [intronic in $M L X$ interacting protein $(M L X I P)$ ] with decreased [odds ratio $(\mathrm{OR})=0.835, P=3.02 \times 10^{-8}$ ] and increased $\left(\mathrm{OR}=1.202, P=3.67 \times 10^{-8}\right)$ the risk of gout, respectively (Table 1).

Having proceeded on the assumption that the nearest genes to the identified SNPs were likely candidates for causality, our results strongly support the associations of $F G F 5$ and MLXIP with the risk of gout, a urate-related disease. These findings agree with those of recent studies, including our own, which identified $F G F 5$ as a serum urate-affecting gene [3]. In a previous trans-ancestry GWAS of serum urate in 457,690 individuals (including subjects with European ancestry, East Asian ancestry, African Americans, South Asian ancestry, and Hispanics) [4], near loci of the two SNPs we herein focused on (rs10857147 and rs 148015593 of which the nearest genes are FGF5 and MLXIP, respectively) were found to be associated with serum urate. The previous study also calculated the gout ORs of their effectallele $\{$ rs $10857147, \mathrm{OR}=1.04$ [95\% confidence interval (CI), 1.01-1.07]; rs148015593, OR $=1.06$ (95\% CI, 1.04-1.09)\}; however, their effects on the risk of gout have hitherto been unclear. We herein provide the first genetic evidence to suggest the pathophysiological importance of $M L X I P$ in the context of gout. MLXIP encodes glucosesensitive transcription factor, which is involved in energy metabolism, including the activation of the pentose phosphate pathways [5] that stimulates de novo purine nucleotide synthesis. Genetic variations in MLXIP may thus influence the endogenous production of uric acid.

Interestingly, although information on blood pressure was not available in this study, the SNP rs 16998073 (upstream of $F G F 5$ ) was reportedly associated with hypertension susceptibility in East Asians [6], in addition to its association with gout as found in this study. Of note, whereas the rs16998073-T allele was associated with a lower risk of gout as shown here, the minor allele (rs16998073-T) is reportedly associated with increased risk of hypertension. These relationships are seemingly paradoxical, given that the elevation of serum urate levels has been thought to be a potential cause of the development of hypertension (although this causality is not conclusive: some Mendelian randomization studies do not support a causal role of serum urate in hypertension [7]). However, such cases can occur, since the influences of a genetic variation on metabolic syndrome components are not always entirely positive or negative. 
For example, despite a positive association with higher triglyceride levels and the risk of dyslipidemia [8] as well as gout [1], an SNP (rs1260326) in the glucokinase regulator (GCKR) gene is reportedly protective against type 2 diabetes $[8,9]$, suggesting the presence of a complex relationship between the components of this metabolic syndrome and their genetic influencers. Hence, via extremely different (independent) molecular bases, genetic variation in FGF5 may influence the risk of gout and hypertension. There is as yet little molecular evidence to support the role of FGF5-a secretory signaling protein [10]—in the pathogenesis of hyperuricemia/gout as well as hypertension. To address these open questions, further investigations are needed into how the genetic variation in FGF5 can affect the biological mechanisms related to urate handling or uric acid-mediated inflammatory processes as well as blood pressure.

In conclusion, our results indicate the significant association of FGF5 and MLXIP with gout susceptibility. While further studies are required to clarify this notion, our findings should contribute to a better understanding of the pathophysiology of gout.

Supplementary Information The online version contains supplementary material available at https://doi.org/10.1007/s13577-021-00665-2.

Acknowledgements We express our sincere thanks to all the participants in this study. Our heartfelt gratitude goes to the members of the Japan Multi-Institutional Collaborative Cohort Study (J-MICC Study) for their support. The authors also specially acknowledge N. Hamajima and $\mathrm{H}$. Tanaka for sample collection and their continuous encouragement of our study. We also thank K. Morichika, M. Miyazawa, and M. Seki (National Defense Medical College) for technical assistance. YT is an Excellent Young Researcher in the MEXT Leading Initiative for Excellent Young Researchers. Collaborators: Members of the Japan Gout Genomics Consortium (Japan Gout) are: Yuya Shirai, J-MICC Study Group (principal investigator: Kenji Wakai), Toru Shimizu, Hiroshi Ooyama, Keiko Ooyama, Mitsuo Nagase, Yuji Hidaka, Hiroshi Nakashima, Yutaka Sakurai, and Masashi Tsunoda.

Author contributions SJC and HM conceived and designed the study; YT, YK, AN, and NS assisted with research design; S-JC, YT, YK, W-TL, SS, C-JC, and HM analyzed data; TN and MN performed the statistical analysis; HM organized this collaborative study; YK, MN, AN, TT, KT, KW, YS, NS, CL, YO, and KI provided intellectual input and assisted with the preparation of the manuscript; S-JC, YT and HM wrote the manuscript; S-JC, YT, and YK contributed equally to this work.

Funding The research conducted by the National Defense Medical College was supported by JSPS KAKENHI (Nos. 16H06279 (PAGS), 221S0002, 17H04128, 20H00566, 20K23152, 21H03350, and 21KK0173); the Ministry of Defense of Japan; the Kawano Masanori Memorial Foundation for Promotion of Pediatrics; and the Gout and Uric Acid Foundation of Japan. The research conducted by Nagoya University Graduate School of Medicine was also supported by a JSPS KAKENHI Grant [No. 16H06277 (CoBiA)] and Grants-in-Aid for Scientific Research on Innovative Areas (No. 221S0001) from the Ministry of Education, Culture, Sports, Science and Technology of Japan.

\section{Declarations}

Conflict of interest The authors declare that they have no conflict of interest.

Ethics approval Data and sample collection for the cohorts participating in the present study were approved by the respective research ethics committees (National Defense Medical College; Nagoya University; National University of Kaohsiung). All the studies were performed according to the guidelines of the Declaration of Helsinki.

Informed consent All participants had provided their written informed consent.

Open Access This article is licensed under a Creative Commons Attribution 4.0 International License, which permits use, sharing, adaptation, distribution and reproduction in any medium or format, as long as you give appropriate credit to the original author(s) and the source, provide a link to the Creative Commons licence, and indicate if changes were made. The images or other third party material in this article are included in the article's Creative Commons licence, unless indicated otherwise in a credit line to the material. If material is not included in the article's Creative Commons licence and your intended use is not permitted by statutory regulation or exceeds the permitted use, you will need to obtain permission directly from the copyright holder. To view a copy of this licence, visit http://creativecommons.org/licenses/by/4.0/.

\section{References}

1. Nakayama A, Nakatochi M, Kawamura Y, et al. Subtype-specific gout susceptibility loci and enrichment of selection pressure on ABCG 2 and ALDH2 identified by subtype genome-wide metaanalyses of clinically defined gout patients. Ann Rheum Dis. 2020;79:657-65.

2. Chen CJ, Tseng CC, Yen JH, et al. ABCG2 contributes to the development of gout and hyperuricemia in a genome-wide association study. Sci Rep. 2018;8:3137.

3. Nakatochi M, Kanai M, Nakayama A, et al. Genome-wide metaanalysis identifies multiple novel loci associated with serum uric acid levels in Japanese individuals. Commun Biol. 2019;2:115.

4. Tin A, Marten J, Halperin Kuhns VL, et al. Target genes, variants, tissues and transcriptional pathways influencing human serum urate levels. Nat Genet. 2019;51:1459-74.

5. Mattila J, Havula E, Suominen E, et al. Mondo-Mlx mediates organismal sugar sensing through the gli-similar transcription factor sugarbabe. Cell Rep. 2015;13:350-64.

6. Xi B, Shen Y, Reilly KH, Wang X, Mi J. Recapitulation of four hypertension susceptibility genes (CSK, CYP17A1, MTHFR, and FGF5) in East Asians. Metabolism. 2013;62:196-203.

7. Johnson RJ, Bakris GL, Borghi C, et al. Hyperuricemia, acute and chronic kidney disease, hypertension, and cardiovascular disease: report of a scientific workshop organized by the national kidney foundation. Am J Kidney Dis. 2018;71:851-65.

8. Vaxillaire M, Cavalcanti-Proenca C, Dechaume A, et al. The common $\mathrm{P} 446 \mathrm{~L}$ polymorphism in GCKR inversely modulates fasting glucose and triglyceride levels and reduces type 2 diabetes risk in the DESIR prospective general French population. Diabetes. 2008;57:2253-7.

9. Matsuo H, Yamamoto K, Nakaoka H, et al. Genome-wide association study of clinically defined gout identifies multiple risk loci and its association with clinical subtypes. Ann Rheum Dis. 2016;75:652-9. 
10. Itoh N, Nakayama Y, Konishi M. Roles of FGFs as paracrine or endocrine signals in liver development, health, and disease. Front Cell Dev Biol. 2016;4:30.
Publisher's Note Springer Nature remains neutral with regard to jurisdictional claims in published maps and institutional affiliations. 